\title{
Matrix formalism for spin dynamics near a single depolarization resonance
}

\author{
Alexander W. Chao \\ Stanford Linear Accelerator Center, Stanford University, Stanford, California 94309, USA
}

(Received 25 July 2005; published 17 October 2005)

\begin{abstract}
A matrix formalism is developed to describe the spin dynamics in a synchrotron near a single depolarization resonance as the particle energy (and therefore its spin precession frequency) is varied in a prescribed pattern as a function of time such as during acceleration. This formalism is first applied to the case of crossing the resonance with a constant crossing speed and a finite total step size, and then applied also to other more involved cases when the single resonance is crossed repeatedly in a prescribed manner consisting of linear ramping segments or sudden jumps. How repeated crossings produce an interference behavior is discussed using the results obtained. For a polarized beam with finite energy spread, a spin echo experiment is suggested to explore this interference effect.
\end{abstract}

PACS numbers: 29.27.Hj, 41.75.-i

\section{INTRODUCTION}

Dynamics of spin motion in a circular accelerator, such as a synchrotron or a storage ring, is conveniently described using a spinor algebra technique [1-6]. If the accelerator has a planar geometry with its nominal bending magnetic field in the vertical $y$ direction, the particle spin will precess rapidly around the $\hat{y}$ axis. This nominal spin precession frequency is equal to $G \gamma$ times the particle's orbital revolution frequency, where $G$ is a fundamental property of the particle given by $G=(g-2) / 2$ with $g$ the gyromaetgnic ratio of the particle, and $\gamma$ is the particle's energy in units of its rest mass. The quantity $G \gamma$ is called the spin tune of the particle. In the case of a synchrotron, as the particle is being accelerated by a constant rate, its spin tune increases linearly with time.

Spin motion is strongly affected if the orbital motion of the particle in the circular accelerator experiences field deviations from the nominal bending fields, and if its spin tune is near a depolarization resonance

$$
G \gamma \approx \kappa
$$

where $\kappa$ specifies the resonance location (for example, $\kappa=$ integer for imperfection resonances, $\kappa=$ integer \pm vertical betatron tune for intrinsic resonances, and $\kappa=$ higher betatron tune side bands for higher order depolarization resonances). In this situation, the perturbation on spin motion can be characterized by a single complex quantity, the resonance strength $\epsilon$, which can be expressed as an appropriate Fourier harmonic of the field deviations around the accelerator [1-4,6,7]. Given the depolarization resonance strength $\epsilon$, as will be assumed in this paper, a question arises as to how the spin motion depends on the pattern in which the spin tune is varied with time in the neighborhood of condition (1). For example, one would be interested in knowing what happens to the spin as the particle is accelerated through a depolarization resonance.

One such analysis has been performed by Froissart and Stora [7], yielding the well-known Froissart-Stora formula that relates the spin polarizations after and before crossing the resonance. The pattern assumed in the Froissart-Stora formula is that of a linear rise of spin tune from $-\infty$ to $+\infty$ with a given constant rate of change.

In practice and in experimental studies of spin dynamics [8], sometimes it would be useful to calculate other patterns of resonance crossing. One example is to start with a spin tune a finite distance (instead of $-\infty$ ) below the resonance, cross the resonance linearly with time, and then park the spin tune a finite distance (instead of $+\infty$ ) above the resonance after crossing. Another example could be crossing the resonance back and forth multiple times linearly in time, and park the spin tune at some location after the process. Still another possibility is to cross the resonance by a sudden jump of spin tune. To study these patterns will require an extension of the Froissart-Stora analysis. In this paper, we offer a matrix formalism based on spinor algebra that allows calculation for spin tune patterns that consist of an arbitrary combination of piecewise constant, piecewise linear, and sudden jumps. It is suggested that this matrix formalism can be used to treat practical experimental conditions beyond the FroissartStora case. Cases thus made calculable offer a range of potentially interesting, and sometimes unexpected, behavior to be studied experimentally.

Note that spin dynamics of a sudden spin tune jump and that of a linear ramping of spin tune over a finite step have both been available in the literature [1-4,6,7]. The matrix formalism proposed here allows combining these results for various practical applications.

Several example applications will be described in subsequent sections. In applications that include multiple resonance crossings, we will explore the constructive and destructive interference effects of these crossings. A condition for destructive interference between two crossings, for example, might be useful if one wishes to compensate a particularly strong depolarization resonance by another artificially induced resonance. 
Experimentally, these interference effects are expected to be most readily observable when the polarized beam has a small energy spread. Ways to produce a beam with small energy spread should help greatly the exploration of these effects. For a polarized beam of larger energy spreads, these effects are not readily observable but an echo experiment is suggested. In this echo experiment, two resonance crossings normally considered to be far separated can still interference with each other to produce an echo at an unexpected long time after the crossings.

\section{EQUATION OF MOTION}

We assume the spin dynamics is determined by one and only one depolarization resonance of strength $\epsilon$ at $G \gamma=$ $\kappa$. We assume $G \gamma$ is near the resonance and its distance to the resonance is a prescribed function of time. In particular, it may occur that the spin tune crosses the resonance with some prescribed manner.

In spinor notation, the spin state of a particle is described by a two-component complex spinor $\psi$, and the spin dynamics is described by

$$
\frac{d \psi}{d \theta}=-\frac{i}{2}\left[\begin{array}{cc}
-G \gamma & \epsilon e^{i \kappa \theta} \\
\epsilon^{*} e^{-i \kappa \theta} & G \gamma
\end{array}\right] \psi,
$$

where $\theta$ is the time variable (advancing by $2 \pi$ per revolution of the particle around the circular accelerator), $G \gamma$ depends on $\theta$ as

$$
G \gamma(\theta)=\kappa+\alpha(\theta)
$$

where $\alpha(\theta)$ is prescribed by the way the resonance is approached or crossed. This spinor equation has implicitly assumed that $\alpha$ and $\epsilon$ as functions of time vary slowly compared to spin precession. This is a good assumption because spin precession is fast. Spin diffusion effects are ignored.

We define

$$
\beta(\theta)=\int_{\theta_{0}}^{\theta} d \theta^{\prime} \alpha\left(\theta^{\prime}\right)
$$

where $\theta_{0}$ refers to the initial time when the spin dynamics is launched, and make the transformation

$$
\psi_{1}=e^{-i / 2[\kappa \theta+\beta(\theta)] \sigma_{y}} \psi
$$

with Pauli matrices

$$
\sigma_{x}=\left[\begin{array}{cc}
0 & -i \\
i & 0
\end{array}\right], \quad \sigma_{y}=\left[\begin{array}{cc}
1 & 0 \\
0 & -1
\end{array}\right], \quad \sigma_{z}=\left[\begin{array}{ll}
0 & 1 \\
1 & 0
\end{array}\right] .
$$

It can be shown that

$$
\frac{d \psi_{1}}{d \theta}=-\frac{i}{2}\left[\begin{array}{cc}
0 & \epsilon(\theta) e^{-i \beta(\theta)} \\
\epsilon^{*}(\theta) e^{i \beta(\theta)} & 0
\end{array}\right] \psi_{1} .
$$

We then let

$$
\psi_{1}(\theta)=\left[\begin{array}{c}
f(\theta) \\
g(\theta) e^{i \beta(\theta)}
\end{array}\right]
$$

to obtain

$$
f^{\prime}=-\frac{i \epsilon}{2} g \quad g^{\prime}=-i \alpha g-\frac{i \epsilon^{*}}{2} f
$$

where a prime designates $d / d \theta$.

It follows from Eq. (8) that

$$
|f|^{2}+|g|^{2}=\text { constant in time } \theta
$$

because $\left(|f|^{2}+|g|^{2}\right)^{\prime}$ can be easily shown to vanish. The initial conditions $f\left(\theta_{0}\right)$ and $g\left(\theta_{0}\right)$ are normalized so that $\left|f\left(\theta_{0}\right)\right|^{2}+\left|g\left(\theta_{0}\right)\right|^{2}=1$. As a result,

$$
|f(\theta)|^{2}+|g(\theta)|^{2}=1 \text { for all } \theta \text {. }
$$

This condition holds even when $\alpha$ and $\epsilon$ are functions of $\theta$.

We will primarily be interested in $P_{y}$, the $y$ component of polarization, in this planar accelerator. This $y$ component at any time $\theta$ is given by

$$
\begin{aligned}
P_{y}(\theta) & =\psi^{\dagger} \sigma_{y} \psi=\psi_{1}^{\dagger} \sigma_{y} \psi_{1}=|f(\theta)|^{2}-|g(\theta)|^{2} \\
& =2|f(\theta)|^{2}-1 .
\end{aligned}
$$

It is sometime useful to eliminate $g$ from Eq. (8), which yields

$$
f^{\prime \prime}(\theta)+\left[i \alpha(\theta)-\frac{\epsilon^{\prime}(\theta)}{\epsilon(\theta)}\right] f^{\prime}(\theta)+\frac{|\epsilon|^{2}}{4} f(\theta)=0 .
$$

Note that, for the case when $\epsilon$ is constant in time, it is $|\epsilon|^{2}$ that plays the role in determining the polarization; the phase of $\boldsymbol{\epsilon}$ does not matter.

We still need to specify the initial conditions of the spin at time $\theta=\theta_{0}$ (one could mentally set $\theta_{0}$ to be a large negative value). Let us designate $\alpha_{0}=\alpha\left(\theta_{0}\right)$ and $\epsilon_{0}=$ $\epsilon\left(\theta_{0}\right)$. We assume that these parameters have been held at these values, and that the spin has been in an eigenstate, from $\theta=-\infty$ until $\theta=\theta_{0}$. The initial spin eigenstate is given by

$$
\left[\begin{array}{l}
f \\
g
\end{array}\right]_{\theta_{0}}=\sqrt{\frac{\Omega+\left|\alpha_{0}\right|}{2 \Omega}}\left[\begin{array}{c}
1 \\
-\left[\operatorname{sgn}\left(\alpha_{0}\right) / \epsilon_{0}\right]\left(\Omega-\left|\alpha_{0}\right|\right)
\end{array}\right],
$$

where

$$
\Omega=\sqrt{\alpha_{0}^{2}+\left|\epsilon_{0}\right|^{2}} .
$$

The initial polarization of this eigenstate is

$$
P_{y}\left(\theta_{0}\right)=\left|f\left(\theta_{0}\right)\right|^{2}-\left|g\left(\theta_{0}\right)\right|^{2}=\frac{\left|\alpha_{0}\right|}{\Omega},
$$

which approaches $100 \%$ when $\left|\alpha_{0}\right| \gg\left|\epsilon_{0}\right|$ as one would expect because the spin tune is far from the resonance. 
Note that being in a pure eigenstate, the spin is initially $100 \%$ polarized although its $y$ component $\left|P_{y}\left(\theta_{0}\right)\right|<1$. Indeed, one may compute the $x$ and $z$ components of the polarization to obtain

$$
\begin{aligned}
& P_{x}\left(\theta_{0}\right)=\frac{\operatorname{sgn}\left(\alpha_{0}\right)}{\Omega} \operatorname{Im}\left(\epsilon_{0} e^{i \kappa \theta_{0}}\right) \\
& P_{z}\left(\theta_{0}\right)=-\frac{\operatorname{sgn}\left(\alpha_{0}\right)}{\Omega} \operatorname{Re}\left(\epsilon_{0} e^{i \kappa \theta_{0}}\right)
\end{aligned}
$$

and $P_{x}^{2}\left(\theta_{0}\right)+P_{z}^{2}\left(\theta_{0}\right)+P_{y}^{2}\left(\theta_{0}\right)=1$.

\section{CONSTANT $\alpha$ AND $\epsilon$}

We are now ready to treat some special cases. Consider the case when $\alpha$ and $\epsilon$ stay at constant values from time $\theta_{1}$ to $\theta_{2}$, i.e., when

$$
\alpha(\theta)=\alpha_{0}, \quad \epsilon(\theta)=\epsilon_{0} .
$$

The spin motion in this period can be written in a matrix form,

$$
\left[\begin{array}{l}
f \\
g
\end{array}\right]_{\theta_{2}>\theta>\theta_{1}}=T_{\alpha_{0}, \epsilon_{0}}\left(\theta, \theta_{1}\right)\left[\begin{array}{l}
f \\
g
\end{array}\right]_{\theta_{1}}
$$

where the map that brings the spin state from $\theta_{1}$ to $\theta$ is designated by a matrix $T_{\alpha_{0}, \epsilon_{0}}\left(\theta, \theta_{1}\right)$. In this simple case, it is given by solving Eq. (8),

$$
\begin{aligned}
T_{\alpha_{0}, \epsilon_{0}}\left(\theta, \theta_{1}\right)= & e^{-(1 / 2) \alpha_{0}\left(\theta-\theta_{1}\right)}\left[\begin{array}{cc}
1 & 0 \\
\alpha_{0} / \epsilon_{0} & i \Omega / \epsilon_{0}
\end{array}\right] \\
& \times\left[\begin{array}{cc}
\cos \Theta & \sin \Theta \\
-\sin \Theta & \cos \Theta
\end{array}\right]\left[\begin{array}{cc}
1 & 0 \\
i \alpha_{0} / \Omega & -i \epsilon_{0} / \Omega
\end{array}\right]
\end{aligned}
$$

where

$$
\Theta=\frac{\Omega\left(\theta-\theta_{1}\right)}{2} .
$$

One can check that the normalization is preserved with this map, i.e., $|f(\theta)|^{2}+|g(\theta)|^{2}=\left|f\left(\theta_{1}\right)\right|^{2}+\left|g\left(\theta_{1}\right)\right|^{2}$.

The special case when $\epsilon_{0}=0$ gives

$$
T_{\alpha_{0}, 0}\left(\theta, \theta_{1}\right)=\left[\begin{array}{cc}
1 & 0 \\
0 & e^{-i \alpha_{0}\left(\theta-\theta_{1}\right)}
\end{array}\right] .
$$

Another special case occurs when $\Theta=m \pi$ where $m$ is an integer, i.e., when the accumulated spin precession angle $\Omega\left(\theta-\theta_{1}\right)$ is an integral multiple of $2 \pi$. Then

$$
T_{\alpha_{0}, \epsilon_{0}}\left(\theta, \theta_{1}\right)=(-1)^{m} e^{-i\left(\alpha_{0} / \Omega\right) m \pi}\left[\begin{array}{ll}
1 & 0 \\
0 & 1
\end{array}\right] .
$$

Other than an overall phase factor, the spin motion in this period is a unit transformation. This period from $\theta_{1}$ to $\theta$ is therefore spin transparent, and has no net effect on the spin motion. Still another special case is when $\Theta=\left(m+\frac{1}{2}\right) \pi$, in which case we have

$$
\begin{aligned}
T_{\alpha_{0}, \epsilon_{0}}\left(\theta, \theta_{1}\right)= & (-1)^{m} e^{-i\left(\alpha_{0} / 2 \Omega\right)(2 m+1) \pi} \frac{1}{\Omega} \\
& \times\left[\begin{array}{cc}
i \alpha_{0} & -i \epsilon_{0} \\
-i \epsilon_{0}^{*} & -i \alpha_{0}
\end{array}\right] .
\end{aligned}
$$

\section{A. Application 1}

As a first application of this case of constant $\alpha$ and constant $\epsilon$, consider a spin launched at time $\theta_{0}$ with initial conditions (13), and let $\alpha$ and $\epsilon$ be kept at their initial values $\alpha_{0}$ and $\epsilon_{0}$. The spin will stay in the eigenstate, with

$$
\begin{aligned}
{\left[\begin{array}{l}
f \\
g
\end{array}\right]_{\theta>\theta_{0}} } & =T_{\alpha_{0}, \epsilon_{0}}\left(\theta, \theta_{0}\right)\left[\begin{array}{l}
f \\
g
\end{array}\right]_{\theta_{0}} \\
& =e^{i / 2\left(\theta-\theta_{0}\right)\left[\operatorname{sgn}\left(\alpha_{0}\right) \Omega-\alpha_{0}\right]}\left[\begin{array}{l}
f \\
g
\end{array}\right]_{\theta_{0}} .
\end{aligned}
$$

In this case, polarization in this time period is preserved,

$$
P_{y}(\theta)=|f(\theta)|^{2}-|g(\theta)|^{2}=\left|f\left(\theta_{0}\right)\right|^{2}-\left|g\left(\theta_{0}\right)\right|^{2}=\frac{\left|\alpha_{0}\right|}{\Omega} .
$$

\section{B. Application 2}

As a second application, consider the case when the resonance strength $\epsilon$ stays constant at the value $\epsilon_{0}$, while the spin tune crosses the resonance at time $\theta=0$ according to a tune-jump pattern,

$$
\alpha(\theta)= \begin{cases}-A, & \text { if } \theta<0 \\ A, & \text { if } \theta>0\end{cases}
$$

where the sign is such that the resonance is crossed from below when $A>0$ and from above if $A<0$. In order for the single-resonance approximation, i.e., Eq. (2), to hold, the sudden change of $\alpha$ is assumed to take place over at least several spin precession periods. At the same time, it should be fast enough to qualify for a sudden jump. These conditions together require that the time during which the jump takes place, measured in number of turns $n_{\text {jump }}$, should satisfy $1 / G \gamma \ll n_{\text {jump }} \ll 1 / \Omega$, where $\Omega$ is to be defined in Eq. (27).

The spin state after crossing is given by

$$
\left[\begin{array}{l}
f \\
g
\end{array}\right]_{\theta>0}=T_{A, \epsilon_{0}}(\theta, 0) T_{-A, \epsilon_{0}}\left(0, \theta_{0}\right)\left[\begin{array}{l}
f \\
g
\end{array}\right]_{\theta_{0}} .
$$

When the initial conditions are specified by (13), then

$$
\left[\begin{array}{l}
f \\
g
\end{array}\right]_{\theta>0}=e^{-(i / 2) A\left(\theta+\theta_{0}\right)} e^{-(i / 2) \operatorname{sgn}(A) \Omega\left(\theta-\theta_{0}\right)} \sqrt{\frac{\Omega+|A|}{2 \Omega}}\left[\begin{array}{c}
1+2 i(A / \Omega) \sin (\Omega \theta / 2) e^{(i / 2) \operatorname{sgn}(A) \Omega \theta} \\
\frac{\operatorname{sgn}(A)}{\epsilon_{0}}(\Omega-|A|)\left[1-2 i(A / \Omega) \sin (\Omega \theta / 2) e^{(i / 2) \operatorname{sgn}(A) \Omega \theta}\right]
\end{array}\right],
$$

where $\Omega=\sqrt{A^{2}+\left|\epsilon_{0}\right|^{2}}$. 
Again one can verify that the normalization $|f(\theta)|^{2}+$ $|g(\theta)|^{2}=1$ holds. The polarization after the spin tune jump is found to be $[1,9]$

$$
\begin{aligned}
P_{y}(\theta>0) & =2|f(\theta)|^{2}-1 \\
& =\frac{|A|\left(A^{2}-\left|\epsilon_{0}\right|^{2}\right)}{\left(A^{2}+\left|\epsilon_{0}\right|^{2}\right)^{3 / 2}}+\frac{2|A|\left|\epsilon_{0}\right|^{2}}{\left(A^{2}+\left|\epsilon_{0}\right|^{2}\right)^{3 / 2}} \cos \Omega \theta .
\end{aligned}
$$

This polarization $P_{y}$ oscillates in time $\theta$ around the mean value $\left[|A|\left(A^{2}-\left|\epsilon_{0}\right|^{2}\right)\right] /\left(A^{2}+\left|\epsilon_{0}\right|^{2}\right)^{3 / 2}$ with frequency $\Omega$, and it oscillates between the maximum value $|A| /\left(A^{2}+\right.$ $\left.\left|\epsilon_{0}\right|^{2}\right)^{1 / 2}$ and the minimum value $|A|\left(A^{2}-3\left|\epsilon_{0}\right|^{2}\right) /\left(A^{2}+\right.$ $\left.\left|\epsilon_{0}\right|^{2}\right)^{3 / 2}$, where the maximum value is actually equal to the initial polarization at time $\theta_{0}$. Note that although the final polarization depends on the observation time $\theta$, it does not depend on the initial time $\theta_{0}$ because the spin was launched in its eigenstate.

\section{Application 3}

As a third application, consider an experiment that turns off the resonance after a tune jump,

$$
\begin{aligned}
& \alpha(\theta)= \begin{cases}-A & \text { if } \theta<0, \\
A, & \text { if } \theta>0,\end{cases} \\
& \epsilon(\theta)=\left\{\begin{array}{ll}
\epsilon_{0}, & \text { if } \theta<\theta_{1} \\
0, & \text { if } \theta>\theta_{1} .
\end{array} \quad\left(\theta_{1}>0\right),\right.
\end{aligned}
$$

The spin state after switching off the resonance is

$$
\left[\begin{array}{l}
f \\
g
\end{array}\right]_{\theta>\theta_{1}}=T_{A, 0}\left(\theta, \theta_{1}\right) T_{A, \epsilon_{0}}\left(\theta_{1}, 0\right) T_{-A, \epsilon_{0}}\left(0, \theta_{0}\right)\left[\begin{array}{l}
f \\
g
\end{array}\right]_{\theta_{0}} .
$$

One can then calculate the polarization $P_{y}\left(\theta>\theta_{1}\right)$ and find that it does not depend on $\theta$ after resonance is switched off, and is given by Eq. (28) with $\theta$ set to $\theta_{1}$, as one might expect.

\section{Application 4}

In this application, we keep the resonance strength $\epsilon_{0}$ fixed, but make a spin tune jump across the resonance at time $\theta=0$, and jump across the resonance back again at time $\theta_{1}>0$, i.e.,

$$
\alpha(\theta)= \begin{cases}-A, & \text { if } \theta<0, \\ A, & \text { if } 0<\theta<\theta_{1}, \\ -A, & \text { if } \theta_{1}<\theta,\end{cases}
$$

and as seen in Fig. 1.

This example is of interest to study the interference effect of two resonance crossings. In general, one may consider crossing two nearby resonances in one tune ramping process. In this case, we try to gain some insight by considering crossing a single resonance twice. In this example, the crossings are performed by sudden tune

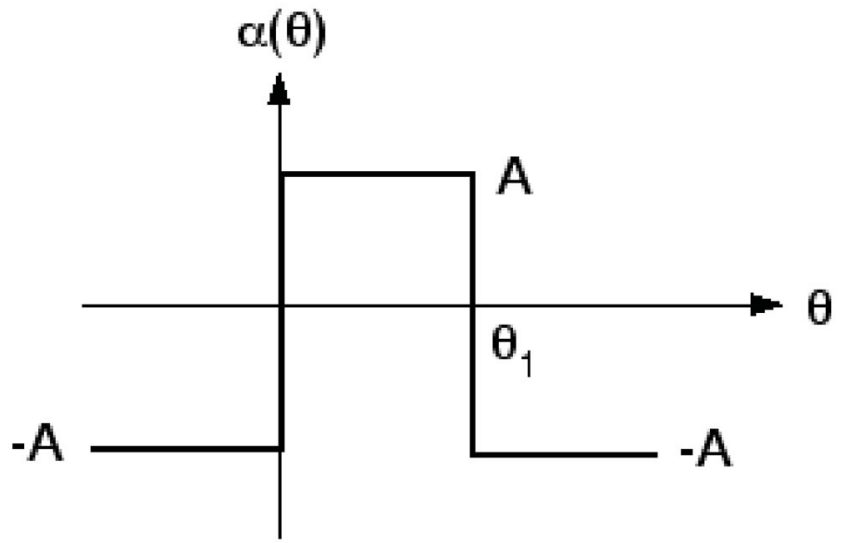

FIG. 1. Spin tune distance to resonance $\alpha$ as a function of time $\theta$ jumping across the resonance twice.

jumps forward and backward, and the two crossings are separated in time by $\theta_{1}$.

The spin state after the second crossing, $\theta>\theta_{1}$, is given by

$$
\left[\begin{array}{l}
f \\
g
\end{array}\right]_{\theta>\theta_{1}}=T_{-A, \epsilon_{0}}\left(\theta, \theta_{1}\right) T_{A, \epsilon_{0}}\left(\theta_{1}, 0\right) T_{-A, \epsilon_{0}}\left(0, \theta_{0}\right)\left[\begin{array}{l}
f \\
g
\end{array}\right]_{\theta_{0}} .
$$

Polarization is then found to be, after some algebra,

$$
\begin{aligned}
P_{y}(\theta)= & \frac{|A|}{\Omega^{5}}\left\{\left[\left(\Omega^{2}-2 A^{2}\right)^{2}+4 A^{2}\left(\Omega^{2}-A^{2}\right) \cos \Omega \theta_{1}\right]\right. \\
& +2\left(\Omega^{2}-A^{2}\right)\left(\Omega^{2}+2 A^{2} \cos \Omega \theta_{1}\right) \\
& \times \cos \Omega \theta\left(1-\cos \Omega \theta_{1}\right)-2\left(\Omega^{2}-A^{2}\right) \\
& \left.\times \sin \Omega \theta \sin \Omega \theta_{1}\left[\Omega^{2}-2 A^{2}\left(1-\cos \Omega \theta_{1}\right)\right]\right\} .
\end{aligned}
$$

The second and third terms of Eq. (32) combine to give an oscillatory contribution $P_{\text {osc }}$ of the polarization after the double spin tune jumps. The oscillation frequency is $\Omega$. The average polarization $P_{\mathrm{av}}$ is given by the first term of Eq. (32). We see that $P_{\text {osc }}$ and $P_{\text {av }}$ depend on $\theta_{1}$, the time separation between the two jumps.

\section{E. Interference and spin echo}

Here we continue the previous application and explore the interference between the two jump crossings and suggest an echo phenomenon to be explored experimentally.

According to Eq. (32), a destructive interference between the two crossings will yield a maximum average polarization, which occurs with

$$
\begin{gathered}
\Omega \theta_{1}=\text { even multiple of } \pi, \\
P_{\mathrm{av}}=\frac{|A|}{\Omega}, \\
P_{\mathrm{osc}}=0 .
\end{gathered}
$$


As mentioned earlier, Eq. (33) is the condition when the spin motion between the two spin tune jumps is transparent. When that happens, the two equal and opposite jumps have a complete destructive interference. The final polarization keeps its initial value, and there is no loss of polarization.

If the spin tune jumps not to $A$ but to some other value $A^{\prime}$ for the time period between 0 and $\theta_{1}$, the condition for destructive interference still occurs at $\sqrt{A^{\prime 2}+|\epsilon|^{2}} \theta_{1}=$ $2 m \pi$. This is valid even if $A^{\prime}$ is negative, i.e., the resonance is not actually crossed.

A constructive interference yields a minimum average polarization, which occurs with

$$
\begin{gathered}
\Omega \theta_{1}=\text { odd multiple of } \pi, \\
P_{\mathrm{av}}=\frac{|A|}{\Omega^{5}}\left(\Omega^{4}-8 A^{2} \Omega^{2}+8 A^{4}\right), \\
P_{\mathrm{osc}}=\frac{4|A|}{\Omega^{5}}\left(\Omega^{2}-A^{2}\right)\left(\Omega^{2}-2 A^{2}\right) \cos \Omega \theta .
\end{gathered}
$$

It should be pointed out that this interference behavior is independent of whether $|A|$ is small or large compared with $|\epsilon|$. It also does not matter whether $\theta_{1}$ is small or large as long as $\Omega \theta_{1}$ is an even or odd multiple of $\pi$. In other words, at least in principle, after crossing a resonance, the potential of interfering strongly with a next resonance crossing lasts indefinitely in time, and occurs even when the resonance is crossed "cleanly" in each crossing (meaning $|A| \gg|\epsilon|)$. In an acceleration process, for example, it is not how long ago a resonance has been crossed or how far the spin tune has passed the resonance that determines whether this resonance crossing can be considered an isolated event. The effect of crossing a resonance carries an infinitely long memory.

The above result applies to the case of a single particle. For a beam of particles with, for example, a finite energy spread among the particles, an averaging on our results over the beam's energy distribution will have to be carried out. It is then expected that some of the interference features we observe here will be diluted [10].

More specifically for the double-jump application here, the polarization for an off-energy particle after the first jump will be determined by

$$
\left[\begin{array}{l}
f \\
g
\end{array}\right]_{\theta_{1}>\theta>0}=T_{A+G \gamma \delta, \epsilon_{0}}(\theta, 0) T_{-A+G \gamma \delta, \epsilon_{0}}\left(0, \theta_{0}\right)\left[\begin{array}{l}
f \\
g
\end{array}\right]_{\theta_{0}},
$$

where $\delta=\Delta \gamma / \gamma$ is the relative energy deviation of the particle, while after the second jump, we have

$$
\begin{aligned}
{\left[\begin{array}{l}
f \\
g
\end{array}\right]_{\theta>\theta_{1}}=} & T_{-A+G \gamma \delta, \epsilon_{0}}\left(\theta, \theta_{1}\right) T_{A+G \gamma \delta, \epsilon_{0}} \\
& \times\left(\theta_{1}, 0\right) T_{-A+G \gamma \delta, \epsilon_{0}}\left(0, \theta_{0}\right)\left[\begin{array}{l}
f \\
g
\end{array}\right]_{\theta_{0}} .
\end{aligned}
$$

The beam polarization will then be obtained by integrating the single-particle polarization over the $\delta$ distribution of the beam.

Figure 2 is an illustration of the transition from a single particle to a beam. Parameters used are $\left|\epsilon_{0}\right|=0.08, G \gamma=$ $4, A=0.1$, and $\Omega \theta_{1}=16 \pi$. The red curve shows the polarization $P_{y}$ as a function of $\theta$ for a single onmomentum particle. It exhibits the case of a complete destructive interference. The green and blue curves are what happens to a beam with Gaussian energy distribution around the on-momentum particle with $\mathrm{rms} \sigma_{\delta}=0.1 \%$ and $0.3 \%$, respectively.

For the blue curve, one sees that the polarization after each jump settles down to some equilibrium value, and each jump reduces the polarization level by a factor $\left(A^{2}-\right.$ $\left.\left|\epsilon_{0}\right|^{2}\right) /\left(A^{2}+\left|\epsilon_{0}\right|^{2}\right)=0.22$, which is what is expected if each jump is to be regarded as an isolated event and if the oscillatory term of polarization after a jump is ignored [keeping only the first term in Eq. (28)]. After each jump, the memory of the jump is kept for a time

$$
\theta_{\text {memory }} \sim \frac{\pi \Omega}{A G \gamma \sigma_{\delta}}
$$

which for the parameters of the blue curve gives $\theta_{\text {memory }} \sim$ 300. After a time of $\theta_{\text {memory }}$, the jump can be considered an isolated event.

One should, however, keep in mind that individual particles will remember the jump much longer. This effect provides the possibility of echo phenomena to be explored in a circular accelerator. In the above example, one manifestation of echo shows up as a secondary excitation around $\theta=2 \theta_{1} \approx 785$. Note that there is an echo even when $\theta_{1}$ is much larger than $\theta_{\text {memory }}$.

To show the echo more clearly, Fig. 3 gives another case when the energy spread is increased to $\sigma_{\delta}=0.8 \%, \theta_{1}$ is extended to $\Omega \theta_{1}=41 \pi$ (it does not matter whether the interference is nominally constructive or destructive), and

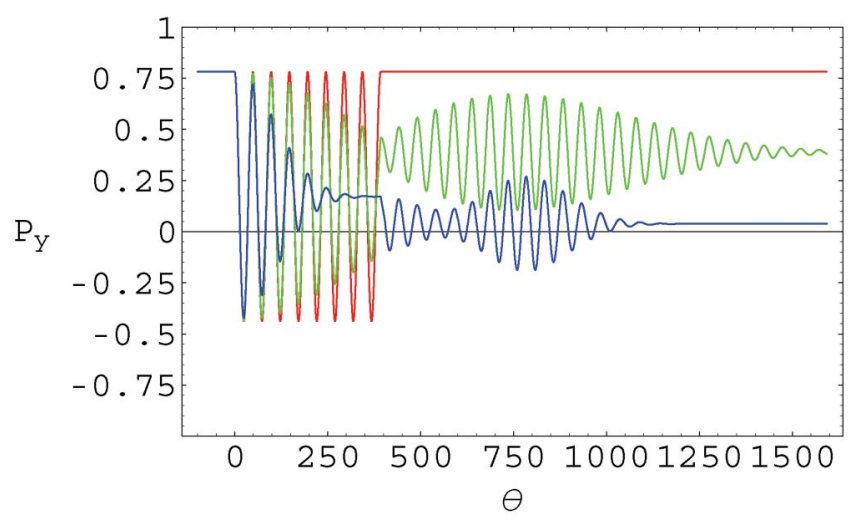

FIG. 2. (Color) Response of a polarized beam to a double-jump across a resonance. Three curves correspond to three different energy spreads of the beam. 


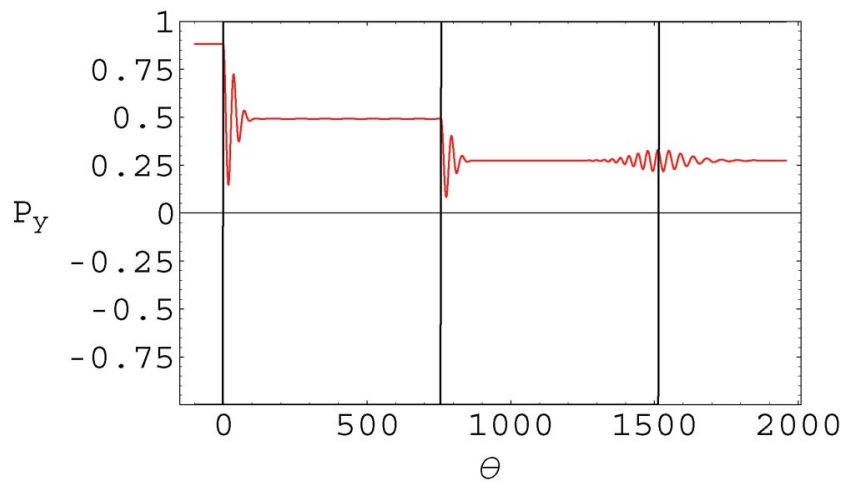

FIG. 3. (Color) An echo effect occurs long time after resonance crossings even when the beam energy spread is large.

$A$ increased to 0.15 . The three vertical black lines indicate the locations of $\theta=0, \theta_{1}, 2 \theta_{1}$. One sees that, although the large energy spread of the beam has apparently completely suppressed any interference effect, there remains an echo of polarization near time $2 \theta_{1}$.

\section{CONSTANT $\epsilon$, LINEAR $\alpha(\theta)$}

In this case, we consider a resonance strength $\epsilon$ that is constant in time, while the spin tune has a linear dependence,

$$
\alpha(\theta)=\left(\theta-\theta_{c}\right) \Gamma
$$

with $\Gamma$ specifying the rate of change of the spin tune ( $\Gamma$ can have either sign) and $\theta_{c}$ specifying the time when spin tune crosses the resonance (by linear extrapolation if resonance is not actually crossed). Solution of spin motion for this linear ramping of spin tune over a finite time period, leading to Eqs. (51) and (52), has been obtained in $[3,4,6,7]$, and Ref. [4] has expressed the result in a matrix form. This result is nevertheless reproduced below for completeness, and will be used as part of the matrix formalism to be developed.

We consider the time range over which Eq. (38) applies to be from $\theta=\theta_{1}$ to $\theta_{2}$. Between $\theta_{1}$ and $\theta_{2}$, we have from Eq. (12)

$$
f^{\prime \prime}(\theta)+i \Gamma\left(\theta-\theta_{c}\right) f^{\prime}(\theta)+\frac{|\epsilon|^{2}}{4} f(\theta)=0 .
$$

Make a transformation

$$
f(\theta)=F(\theta) e^{-i(\Gamma / 4)\left(\theta-\theta_{c}\right)^{2}}
$$

and change variable from $\theta$ to

$$
x=\sqrt{|\Gamma|}\left(\theta-\theta_{c}\right)
$$

then we obtain

$$
\frac{d^{2} F}{d x^{2}}+\left(\frac{x^{2}}{4}-a\right) F=0
$$

where

$$
a=\frac{i}{2} \operatorname{sgn}(\Gamma)-\frac{|\epsilon|^{2}}{4|\Gamma|} .
$$

Note that in this case only the absolute value $|\epsilon|$ enters. (The phase of $\epsilon$ matters only as an overall phase in determining $g$, which does not have physical consequences.) Given $F(\theta)$, polarization is given by $P_{y}(\theta)=$ $2|F(\theta)|^{2}-1$.

Two independent solutions for $F$ can be expressed in terms of the degenerate hypergeometric function ${ }_{1} F_{1}$ $[3,4,7,11]$,

$$
\begin{aligned}
& y_{1}(x)=e_{1}^{-i\left(x^{2} / 4\right)} F_{1}\left(\frac{1}{4}-i \frac{a}{2} ; \frac{1}{2} ; i \frac{x^{2}}{2}\right), \\
& y_{2}(x)=x e_{1}^{i\left(x^{2} / 4\right)} F_{1}\left(\frac{3}{4}+i \frac{a}{2} ; \frac{3}{2} ;-i \frac{x^{2}}{2}\right) .
\end{aligned}
$$

It is useful here to give explicit expressions also for their derivatives,

$$
\begin{aligned}
\frac{d y_{1}}{d x}(x)= & \frac{x}{2} e^{-i\left(x^{2} / 4\right)}\left[-i_{1} F_{1}\left(\frac{1}{4}-i \frac{a}{2} ; \frac{1}{2} ; i \frac{x^{2}}{2}\right)\right. \\
& \left.+(i+2 a)_{1} F_{1}\left(\frac{5}{4}-i \frac{a}{2} ; \frac{3}{2} ; i \frac{x^{2}}{2}\right)\right], \\
\frac{d y_{2}}{d x}(x)= & \frac{1}{6} e^{i\left(x^{2} / 4\right)}\left[3\left(2+i x^{2}\right)_{1} F_{1}\left(\frac{3}{4}+i \frac{a}{2} ; \frac{3}{2} ;-i \frac{x^{2}}{2}\right)\right. \\
& \left.-(3 i-2 a) x^{2}{ }_{1} F_{1}\left(\frac{7}{4}+i \frac{a}{2} ; \frac{5}{2} ;-i \frac{x^{2}}{2}\right)\right] .
\end{aligned}
$$

It is easy to show that the Wronskian

$$
W(x)=y_{1}(x) \frac{d y_{2}}{d x}(x)-\frac{d y_{1}}{d x}(x) y_{2}(x)
$$

is a constant in $x$, and is equal to 1 .

The general solution for $F$ is

$$
F(x)=C_{1} y_{1}(x)+C_{2} y_{2}(x),
$$

where $C_{1,2}$ are determined by the initial conditions. Given $f\left(\theta_{1}\right)$ and $g\left(\theta_{1}\right)$ at initial time $\theta_{1}$, we have the initial conditions, in terms of the $x$ variable,

$$
F\left(x_{1}\right)=f\left(x_{1}\right) e^{(i / 4) \operatorname{sgn}(\Gamma) x_{1}^{2}},
$$

$$
\begin{aligned}
\frac{d F}{d x}\left(x_{1}\right)= & {\left[-\frac{i \epsilon}{2 \sqrt{|\Gamma|}} g\left(x_{1}\right)\right.} \\
& \left.+\frac{i}{2} \operatorname{sgn}(\Gamma) x_{1} f\left(x_{1}\right)\right] e^{(i / 4) \operatorname{sgn}(\Gamma) x_{1}^{2}} .
\end{aligned}
$$


We then have, now expressed in matrix form,

$$
\left[\begin{array}{l}
C_{1} \\
C_{2}
\end{array}\right]=\left[\begin{array}{cc}
\frac{d y_{2}}{d x} & -y_{2} \\
-\frac{d y_{1}}{d x} & y_{1}
\end{array}\right]_{x_{1}} e^{(i / 4) \operatorname{sgn}(\Gamma) x_{1}^{2}}\left[\begin{array}{cc}
1 & 0 \\
\frac{i x_{1}}{2} \operatorname{sgn}(\Gamma) & -\frac{i \epsilon}{2 \sqrt{|\Gamma|}}
\end{array}\right]\left[\begin{array}{l}
f \\
g
\end{array}\right]_{x_{1}}
$$

For time $\theta_{2}>\theta>\theta_{1}$, we have

$$
\left[\begin{array}{c}
F \\
\frac{d F}{d x}
\end{array}\right]_{x}=\left[\begin{array}{cc}
y_{1} & y_{2} \\
\frac{d y_{1}}{d x} & \frac{d y_{2}}{d x}
\end{array}\right]_{x}\left[\begin{array}{l}
C_{1} \\
C_{2}
\end{array}\right]
$$

which then finally yields

$$
\left[\begin{array}{l}
f \\
g
\end{array}\right]_{\theta}=U_{\Gamma, \theta_{c}, \epsilon}\left(\theta, \theta_{1}\right)\left[\begin{array}{l}
f \\
g
\end{array}\right]_{\theta_{1}}
$$

where a map has been defined

$$
U_{\Gamma, \theta_{c}, \epsilon}\left(\theta, \theta_{1}\right)=e^{(i / 4) \operatorname{sgn}(\Gamma)\left(x_{1}^{2}-x^{2}\right)}\left[\begin{array}{cc}
1 & 0 \\
\frac{\operatorname{sgn}(\Gamma) \sqrt{|\Gamma|}}{\epsilon} x & 2 i \frac{\sqrt{|\Gamma|}}{\epsilon}
\end{array}\right]\left[\begin{array}{cc}
y_{1} & y_{2} \\
\frac{d y_{1}}{d x} & \frac{d y_{2}}{d x}
\end{array}\right]_{x}\left[\begin{array}{cc}
\frac{d y_{2}}{d x} & -y_{2} \\
-\frac{d y_{1}}{d x} & y_{1}
\end{array}\right]_{x_{1}}\left[\begin{array}{cc}
1 & 0 \\
\frac{i x_{1}}{2} \operatorname{sgn}(\Gamma) & -\frac{i \epsilon}{2 \sqrt{|\Gamma|}}
\end{array}\right] .
$$

The outer two matrices of the above product bring the $f, g$ representation to $F, d F / d x$, while the middle two matrices describe the dynamics of $F, d F / d x$. When $\theta=\theta_{1}$, we have $U=1$.

\section{A. Application 5}

As an application of the case with linear ramping in spin tune, let us start with initial condition (13) at time $\theta_{0}$, and cross the resonance in a sloped-step pattern,

$$
\alpha(\theta)= \begin{cases}-A, & \text { if } \theta<-\frac{A}{\Gamma} \\ \Gamma \theta, & \text { if } \frac{A}{\Gamma}>\theta>-\frac{A}{\Gamma} \\ A, & \text { if } \theta>\frac{A}{\Gamma}\end{cases}
$$

as shown in Fig. 4.

The crossing speed is specified by the slope $\Gamma$. The spin tune swings from $-A$ to $A$ linearly. We will consider $\Gamma$ and $A$ to have the same sign. When $A>0, \Gamma>0$, the resonance is crossed from below as shown in Fig. 4. When $A<0, \Gamma<$ 0 , the resonance is crossed from above. The following analysis applies to both cases. The resonance strength has a constant value $\epsilon$ throughout the process.

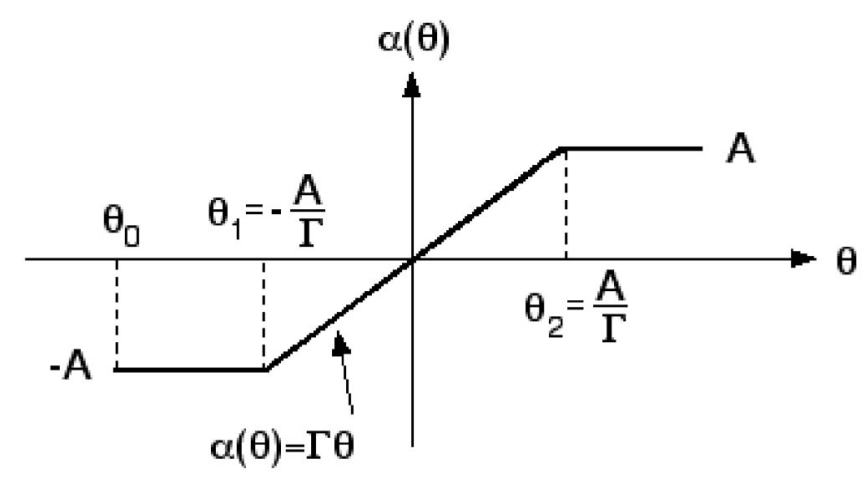

FIG. 4. Crossing a resonance in a sloped-step pattern.
For time $\theta_{1}>\theta>\theta_{0}$ before starting the crossing, spin state is mapped by

$$
\left[\begin{array}{l}
f \\
g
\end{array}\right]_{\theta}=T_{-A, \epsilon}\left(\theta, \theta_{0}\right)\left[\begin{array}{l}
f \\
g
\end{array}\right]_{\theta_{0}}
$$

where $f\left(\theta_{0}\right)$ and $g\left(\theta_{0}\right)$ are given by Eq. (13) with $\alpha_{0}$ and $\epsilon_{0}$ replaced by $-A$ and $\epsilon$ respectively. The result has been given by Eq. (23). The polarization is constant,

$$
P_{y}(\theta)=\frac{|A|}{\sqrt{A^{2}+|\epsilon|^{2}}} .
$$

During the crossing, $\theta_{2}>\theta>\theta_{1}$, the spin state is given by

$$
\left[\begin{array}{l}
f \\
g
\end{array}\right]_{\theta}=U_{\Gamma, 0, \epsilon}\left(\theta,-\frac{A}{\Gamma}\right) T_{-A, \epsilon}\left(-\frac{A}{\Gamma}, \theta_{0}\right)\left[\begin{array}{l}
f \\
g
\end{array}\right]_{\theta_{0}} .
$$

We now define four quantities

$$
\begin{aligned}
& Y_{k}=y_{k}\left(x_{2}\right)=y_{k}\left(\frac{|A|}{\sqrt{|\Gamma|}}\right), \\
& Y_{k}^{\prime}=\frac{d y_{k}}{d x}\left(x_{2}\right)=\frac{d y_{k}}{d x}\left(\frac{|A|}{\sqrt{|\Gamma|}}\right), \quad k=1,2 .
\end{aligned}
$$

Since $y_{1}(x)$ and $\left(d y_{2} / d x\right)(x)$ are even functions of $x, y_{2}(x)$ and $\left(d y_{1} / d x\right)(x)$ are odd functions of $x$, and $x_{1}=-x_{2}$, we have $y_{1}\left(x_{1}\right)=Y_{1}, \quad\left(d y_{1} / d x\right)\left(x_{1}\right)=-Y_{1}^{\prime}, \quad y_{2}\left(x_{1}\right)=-Y_{2}$, $\left(d y_{2} / d x\right)\left(x_{1}\right)=Y_{2}^{\prime}$.

After some algebra, we find by using Eq. (56) that, for $\theta_{2}>\theta>\theta_{1}$, the polarization is given by

$$
\begin{aligned}
P_{y}(\theta)= & \frac{\Omega+|A|}{\Omega} \mid y_{1} Y_{2}^{\prime}+y_{2} Y_{1}^{\prime} \\
& -\left.\frac{i \operatorname{sgn}(A) \Omega}{2 \sqrt{|\Gamma|}}\left(y_{1} Y_{2}+y_{2} Y_{1}\right)\right|^{2}-1,
\end{aligned}
$$


where $y_{1,2}$ are evaluated at $x=\sqrt{|\Gamma|} \theta$ and $\Omega=$ $\sqrt{A^{2}+|\epsilon|^{2}}$.

We then proceed to calculate the spin state after crossing, $\theta>\theta_{2}$,

$$
\begin{aligned}
{\left[\begin{array}{l}
f \\
g
\end{array}\right]_{\theta}=} & T_{A, \epsilon}\left(\theta, \frac{A}{\Gamma}\right) U_{\Gamma, 0, \epsilon}\left(\frac{A}{\Gamma},-\frac{A}{\Gamma}\right) T_{-A, \epsilon}\left(-\frac{A}{\Gamma}, \theta_{0}\right) \\
& \times\left[\begin{array}{c}
f \\
g
\end{array}\right]_{\theta_{0}} .
\end{aligned}
$$

After some algebra, we find

$$
\begin{aligned}
P_{y}(\theta)= & \frac{\Omega+|A|}{\Omega} \mid\left(Y_{1} Y_{2}^{\prime}+Y_{2} Y_{1}^{\prime}\right) e^{-i \operatorname{sgn}(A) \Theta} \\
& -\frac{i \operatorname{sgn}(A) \Omega}{\sqrt{|\Gamma|}} Y_{1} Y_{2} \cos \Theta+\left.\frac{4 \sqrt{|\Gamma|}}{\Omega} Y_{1}^{\prime} Y_{2}^{\prime} \sin \Theta\right|^{2}-1
\end{aligned}
$$

where $\Theta=(\Omega / 2)[\theta-(A / \Gamma)]$. This polarization oscillates in time $\theta$ with frequency $\Omega$. Equation (60) reduces to (28) in the limit $|\Gamma| \rightarrow \infty$, as it should.

Equations (55), (58), and (60) combine to describe the polarization as a function of time during the crossing process. Figure 5 shows $P_{y}(\theta)$ as a function of $\sqrt{\Gamma} \theta$ for 3 cases (with $A>0, \Gamma>0$ ):

$$
\begin{aligned}
& \frac{A}{\sqrt{\Gamma}}=\pi, \quad \frac{|\epsilon|}{\sqrt{\Gamma}}=1 \quad \text { red, } \\
& \frac{A}{\sqrt{\Gamma}}=\pi, \quad \frac{|\epsilon|}{\sqrt{\Gamma}}=4 \quad \text { green, } \\
& \frac{A}{\sqrt{\Gamma}}=1, \quad \frac{|\epsilon|}{\sqrt{\Gamma}}=1 \quad \text { blue. }
\end{aligned}
$$

\section{B. Comparison with Froissart-Stora formula [7]}

Equation (60) may be considered an extension of the Froissart-Stora formula, which was derived assuming the resonance was crossed linearly with an infinite step-size

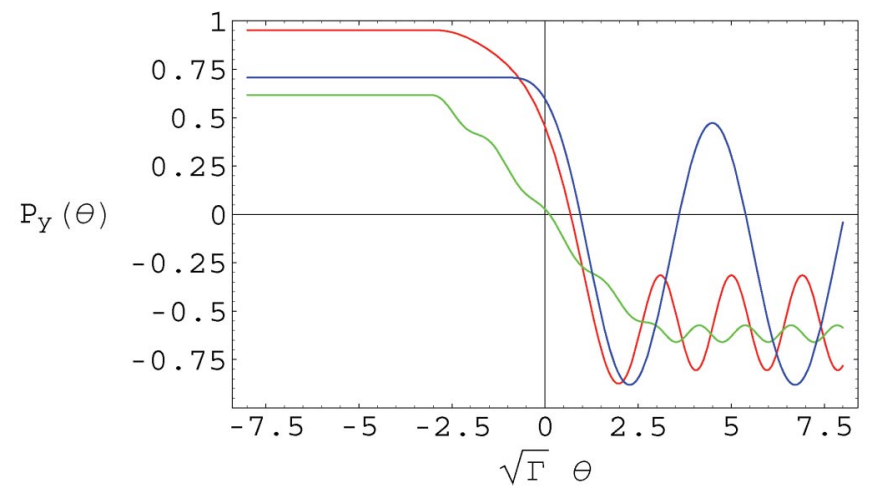

FIG. 5. (Color) Single-particle polarization as function of time during a sloped-step resonance crossing for three example cases. from $\theta=-\infty$ to $\theta=+\infty$. In an experiment with finite step-size, we will consider Eq. (60) to be the "final polarization" after crossing. Unlike the Froissart-Stora formula, this polarization depends on the exact time of observation, and it oscillates sinusoidally with a frequency $\Omega=$ $\sqrt{A^{2}+|\epsilon|^{2}}$ and an amplitude $\pm P_{\text {osc }}= \pm\left|D_{1}^{2}+D_{2}^{2}\right|$, centered around $P_{\mathrm{av}}=\left|D_{1}\right|^{2}+\left|D_{2}\right|^{2}-1$, i.e.

$$
P_{\text {final }}=\left(\left|D_{1}\right|^{2}+\left|D_{2}\right|^{2}-1\right) \pm\left|D_{1}^{2}+D_{2}^{2}\right|
$$

with

$$
\begin{aligned}
& D_{1}=\sqrt{\frac{\Omega+|A|}{2 \Omega}}\left[Y_{1} Y_{2}^{\prime}+Y_{1}^{\prime} Y_{2}-i \frac{\operatorname{sgn}(A) \Omega}{\sqrt{|\Gamma|}} Y_{1} Y_{2}\right], \\
& D_{2}=\sqrt{\frac{\Omega+|A|}{2 \Omega}}\left[\frac{4 \sqrt{|\Gamma|}}{\Omega} Y_{1}^{\prime} Y_{2}^{\prime}-i \sin (A)\left(Y_{1} Y_{2}^{\prime}+Y_{1}^{\prime} Y_{2}\right)\right] .
\end{aligned}
$$

This final polarization depends only on two scaled variables

$$
\bar{A} \equiv \frac{|A|}{2 \sqrt{|\Gamma|}}, \quad \bar{\epsilon} \equiv \frac{|\epsilon|}{2 \sqrt{|\Gamma|}},
$$

where $\bar{A}$ is the normalized step size, $\bar{\epsilon}$ is the normalized resonance width.

The red curves in Fig. 6 are the mean, the upper bound, and the lower bound values of the final polarization as functions of $\bar{\epsilon}$ for four cases $\bar{A}=0.5$ (upper left), 1 (upper right), 2 (lower left), and 10 (lower right). The green curves are the Froissart-Stora formula [7]

$$
P_{\text {final,FS }}=2 e^{-2 \pi \bar{\epsilon}^{2}}-1 .
$$

The Froissart-Stora formula of course does not depend on $\bar{A}$. The green curves in all four subfigures are therefore identical. Naturally, the Froissart-Stora prediction is accurate when $\bar{A} \gg \bar{\epsilon}$ such as the lower right subfigure shows, and it becomes rather inaccurate when $\bar{A}$ becomes comparable or less than $\bar{\epsilon}$ as seen, e.g., in the upper left subfigure.

In an actual spin resonance crossing, it is therefore necessary to confirm first whether the experimental conditions are such that the Froissart-Stora formula is applicable. With the inclusion of the finite step size, our result offers the following observations:

(i) Polarization after crossing differs from FroissartStora formula when $|A| \lesssim|\epsilon|$.

(ii) In general, the final polarization will depend on the time of observation. The oscillation frequency after crossing $\Omega$, if can be measured, offers another way to measure $|\epsilon|$.

(iii) A complete spin flip for large $\bar{\epsilon}$, as predicted by Froissart-Stora formula is modified in such a way that the spin flip efficiency will start to decrease if $\bar{\epsilon}$ approaches $\bar{A}$ [12].

It might be useful to experimentally test some of these observations beyond the Froissart-Stora regime. 

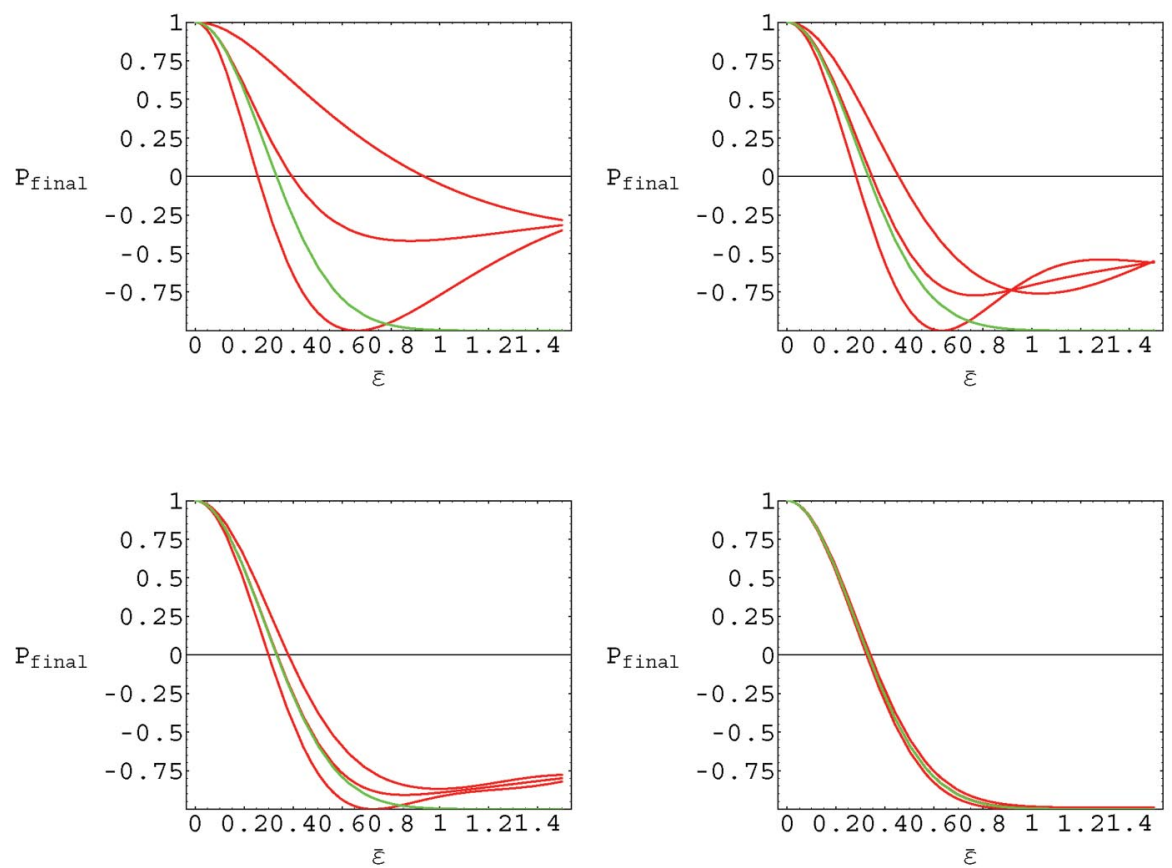

FIG. 6. (Color) Final polarization after crossing a resonance in a sloped-step pattern as function of $\bar{\epsilon}$ for four different values of $\bar{A}$. Red curves give the mean, upper bound, and lower bound of the oscillating final polarization. Green curves are given by the Froissart-Stora formula.

One curiosity occurs when the final polarization does not oscillate under some special conditions of parameters. This occurs when $D_{1}= \pm i D_{2}$ and therefore $P_{\text {osc }}=0$. From Fig. 6, one sees an example when $\bar{A}=1$ and $\bar{\epsilon}=0.915$ where a node point appears. Indeed, a numerical calculation reconfirms this observation.

\section{Application 6: More on interference}

As the next application, let us cross the resonance twice in a back and forth pattern shown in Fig. 7,

$$
\alpha(\theta)= \begin{cases}-A, & \text { if } \theta<-\frac{2 A}{\Gamma}, \\ \Gamma \theta+A, & \text { if } 0>\theta>-\frac{2 A}{\Gamma}, \\ -\Gamma \theta+A, & \text { if } \frac{2 A}{\Gamma}>\theta>0, \\ -A, & \text { if } \theta>\frac{2 A}{\Gamma}\end{cases}
$$

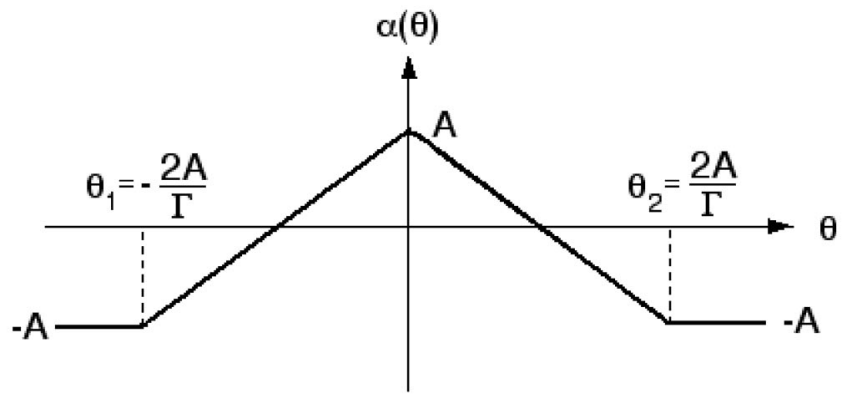

FIG. 7. Double crossing of a resonance in a sloped-step pattern.
We shall try to investigate the interference behavior between the two crossings. We consider the case $A>0$, $\Gamma>0$.

Spin state after crossing $(\theta>2 A / \Gamma)$ is given by

$$
\begin{aligned}
{\left[\begin{array}{l}
f \\
g
\end{array}\right]_{\theta}=} & T_{-A, \epsilon}\left(\theta, \frac{2 A}{\Gamma}\right) U_{-\Gamma, \frac{A}{\Gamma}, \epsilon}\left(\frac{2 A}{\Gamma}, 0\right) U_{\Gamma,-\frac{A}{\Gamma}, \epsilon} \\
& \times\left(0,-\frac{2 A}{\Gamma}\right) T_{-A, \epsilon}\left(-\frac{2 A}{\Gamma}, \theta_{0}\right)\left[\begin{array}{l}
f \\
g
\end{array}\right]_{\theta_{0}} .
\end{aligned}
$$

As before, polarization after the two resonance crossings, given by $2|f|^{2}-1$, oscillates in time $\theta$ with frequency $\Omega=\sqrt{A^{2}+|\epsilon|^{2}}$ around a mean value. It is found to be given by Eq. (61) with

$$
\begin{aligned}
& D_{1}=\sqrt{\frac{\Omega+|A|}{2 \Omega}}\left[\mathcal{M}_{11}-i \mathcal{M}_{12}\right], \\
& D_{2}=\sqrt{\frac{\Omega+|A|}{2 \Omega}}\left[\mathcal{M}_{21}-i \mathcal{M}_{22}\right],
\end{aligned}
$$

where $\mathcal{M}_{i j}$ are the elements of the matrix

$$
\begin{aligned}
\mathcal{M}= & {\left[\begin{array}{cc}
Z_{1} Z_{2}^{\prime}+Z_{2} Z_{1}^{\prime} & 2 \bar{\Omega} Z_{1} Z_{2} \\
(2 / \Omega) Z_{1}^{\prime} Z_{2}^{\prime} & Z_{1} Z_{2}^{\prime}+Z_{2} Z_{1}^{\prime}
\end{array}\right] } \\
& \times\left[\begin{array}{cc}
Y_{1} Y_{2}^{\prime}+Y_{2} Y_{1}^{\prime} & 2 \bar{\Omega} Y_{1} Y_{2} \\
(2 / \bar{\Omega}) Y_{1}^{\prime} Y_{2}^{\prime} & Y_{1} Y_{2}^{\prime}+Y_{2} Y_{1}^{\prime}
\end{array}\right],
\end{aligned}
$$

with $\bar{\Omega}=(\Omega / 2 \sqrt{\Gamma})=\sqrt{\bar{A}^{2}+\bar{\epsilon}^{2}}$. The quantities $Y^{\prime}$ s were defined in Eq. (57), evaluated at $x=(A / \sqrt{\Gamma})=2 \bar{A}$ and 

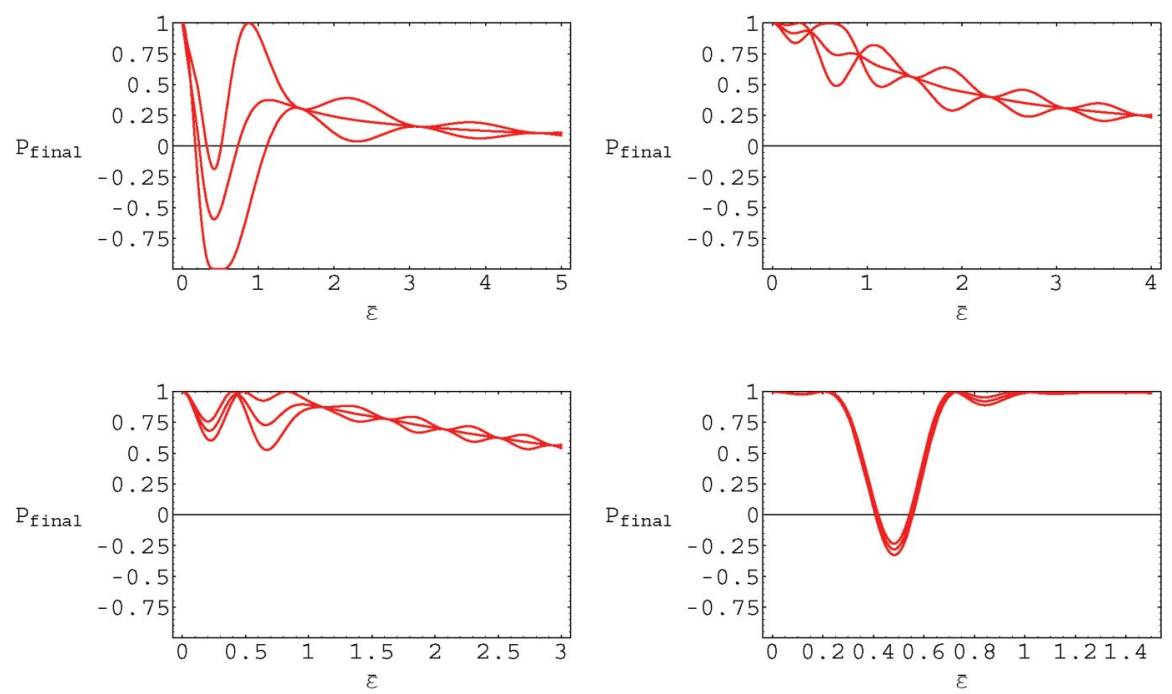

FIG. 8. (Color) Final polarization after twice crossing a resonance in a sloped-step pattern as function of $\bar{\epsilon}$ for four different values of $\bar{A}$. Red curves give the mean, upper bound, and lower bound of the oscillating final polarization.

$a=\frac{i}{2}-\left(|\epsilon|^{2} / 4 \Gamma\right)=\frac{i}{2}-\bar{\epsilon}^{2}$, while $Z$ 's are the same as the $Y$ 's evaluated at the same $x=2 \bar{A}$ but a different $a=$ $-\frac{i}{2}-\bar{\epsilon}^{2}$, where $\bar{A}$ and $\bar{\epsilon}$ were defined in Eq. (63).

Figure 8 shows the mean, the upper bound, and the lower bound values of polarization as a function of $\bar{\epsilon}$, again for four cases $\bar{A}=0.5$ (upper left), 1 (upper right), 2 (lower left), 10 (lower right). A computer code was used to spot check the results by numerically integrating Eq. (12). Agreement was obtained.

Figure 9 shows the mean, the upper bound, and the lower bound values of polarization as a function of $\bar{A}$ for cases $\bar{\epsilon}=0.1$ (upper left), 0.5 (upper right), 1 (lower left).

Figures 8 and 9 exhibit a complex dependence of the polarization on the resonance and spin tune parameters. Here we suggest to look particularly into the interference pattern of the two crossings. One notices the quasiperiodic behavior of the polarization in both $\bar{\epsilon}$ and $\bar{A}$. To explore the condition for the interference pattern, consider the spin precession angle $\Delta \Psi$ accumulated between the two resonance crossings. The exact value of $\Delta \Psi$ has to be found by the hypergeometric functions as our results indicate, but as an approximation, we write

$$
\Delta \Psi \approx \int_{-A / \Gamma}^{A / \Gamma} d \theta \sqrt{\alpha^{2}(\theta)+|\epsilon|^{2}}=8 \int_{0}^{\bar{A}} d x \sqrt{x^{2}+\bar{\epsilon}^{2}}
$$

We then make the following observations from Figs. 8 and 9:
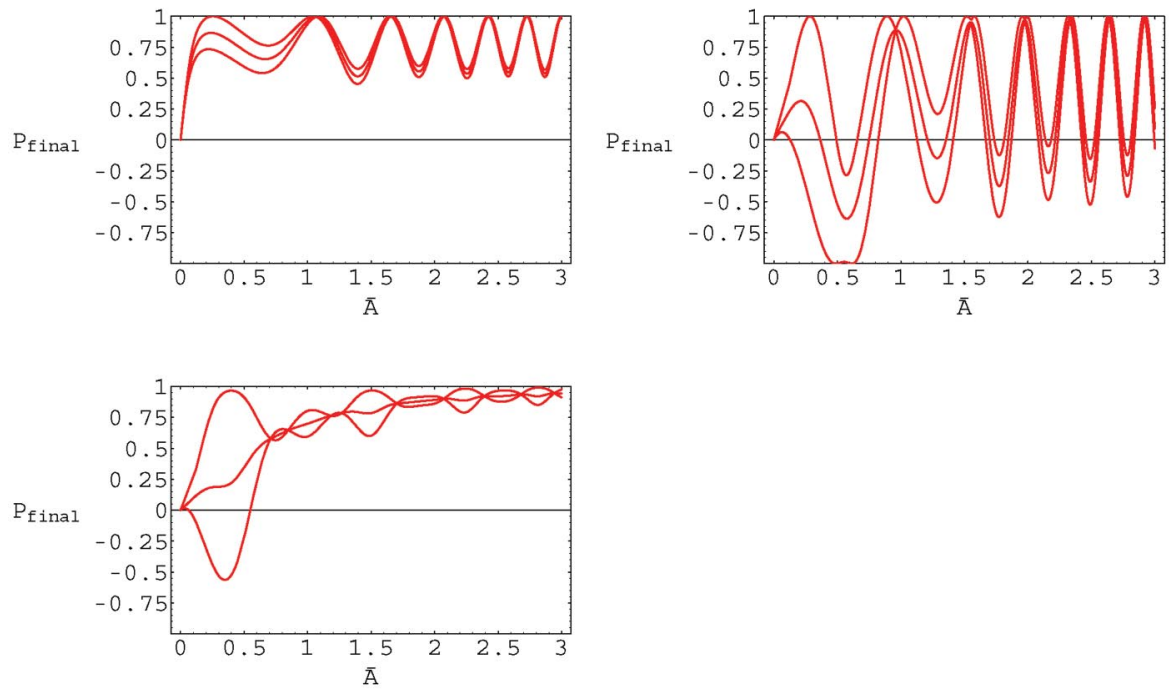

FIG. 9. (Color) Final polarization after twice crossing a resonance in a sloped-step pattern as function of $\bar{A}$ for three different values of $\bar{\epsilon}$. 
(i) Fig. 8 shows the occurrence of nodes when the final polarization ceases to oscillate in time. Following the discussion in Eq. (33), one might anticipate a destructive interference to occur when $\Delta \Psi$ is equal to an even multiple of $\pi$. When $\bar{A} \ll \bar{\epsilon}, \Delta \Psi \approx 8 \bar{\epsilon} \bar{A}$, this means destructive interferences occur when

$$
\bar{\epsilon} \approx \frac{m \pi}{4 \bar{A}}, \quad m=\text { integer. }
$$

This conclusion agrees with the figure. It is further noted that when a destructive interference occurs, not only the final polarization ceases to oscillate, but also it is equal to the initial eigenstate polarization $P_{y}=\bar{A} / \bar{\Omega}$. There is therefore no net depolarization in the double resonance crossings.

(ii) When $\bar{A} \gg \bar{\epsilon}$, it seems from Fig. 9 that destructive nodes occur when $\Delta \Psi$ is equal to an even multiple of $\pi$ although with an apparent small shift that depends on $\bar{\epsilon}$,

$$
\begin{aligned}
& \Delta \Psi \approx \begin{cases}2 \pi(m-0.24), & \text { for } \bar{\epsilon}=0.1, \\
2 \pi(m-0.14), & \text { for } \bar{\epsilon}=0.5, \\
2 \pi(m-0.05), & \text { for } \bar{\epsilon}=1,\end{cases} \\
& m=\text { integer. }
\end{aligned}
$$

(iii) When a constructive interference occurs, one might expect a maximum loss of polarization that to some degree resembles crossing a single resonance of strength $2 \epsilon$. This indeed seems to be the case when $\bar{\epsilon} \ll 1<\bar{A}$, as seen in Fig. 9 with $\bar{\epsilon}=0.1$. The minimum level of polarization is approximately equal to the Froissart-Stora prediction of $2 e^{-2 \pi(2 \bar{\epsilon})^{2}}-1=0.55$. However, this expectation breaks down when $\bar{\epsilon}$ is increased. In fact, when $\bar{\epsilon}$ is increased to become comparable or larger than 1 , the polarization increases contrary to expectation and becomes close to $100 \%$ as seen, for example, in the case $\bar{\epsilon}=1$, indicating the two crossings tend to cancel each other completely regardless of the value of $\bar{A}$, i.e., regardless of how far apart the two crossings might be intended.

It is clear from these observations that the interference behavior of resonance crossings contains some surprises, and exhibits a potentially rich area to be explored.

\section{Application 7}

As the next application, we cross the resonance three times, twice in the same direction, and once by a jump, as shown in Fig. 10,

$$
\alpha(\theta)= \begin{cases}-A, & \text { if } \theta<-\frac{2 A}{\Gamma} \\ \Gamma \theta+A, & \text { if } 0>\theta>-\frac{2 A}{\Gamma} \\ \Gamma \theta-A, & \text { if } \frac{2 A}{\Gamma}>\theta>0 \\ -A, & \text { if } \theta>\frac{2 A}{\Gamma}\end{cases}
$$

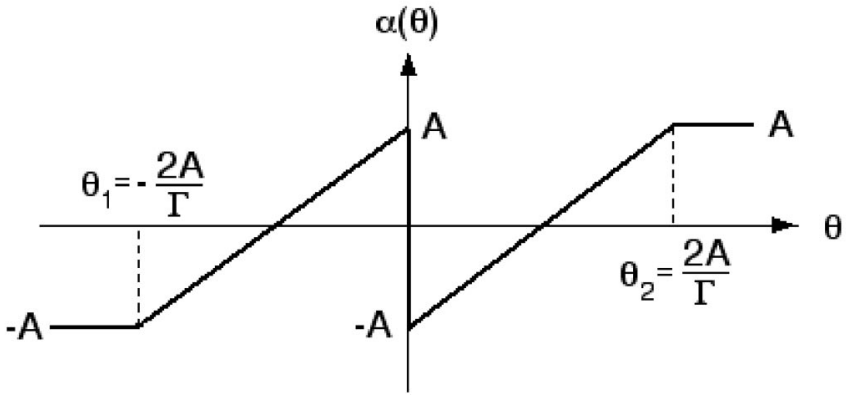

FIG. 10. Crossing the resonance 3 times, two sloped-steps and one sudden jump.

We again assume $A>0, \Gamma>0$. Spin state after crossing $(\theta>2 A / \Gamma)$ is given by

$$
\begin{aligned}
{\left[\begin{array}{l}
f \\
g
\end{array}\right]_{\theta}=} & T_{A, \epsilon}\left(\theta, \frac{2 A}{\Gamma}\right) U_{\Gamma, A / \Gamma, \epsilon}\left(\frac{2 A}{\Gamma}, 0\right) U_{\Gamma,-(A / \Gamma), \epsilon} \\
& \times\left(0,-\frac{2 A}{\Gamma}\right) T_{-A, \epsilon}\left(-\frac{2 A}{\Gamma}, \theta_{0}\right)\left[\begin{array}{l}
f \\
g
\end{array}\right]_{\theta_{0}} .
\end{aligned}
$$

The final polarization again oscillates according to Eq. (61), where $D_{1,2}$ are given by Eq. (67) but the matrix $\mathcal{M}$ of Eq. (68) is replaced by

$$
\begin{aligned}
\mathcal{M}= & {\left[\begin{array}{cc}
Y_{1} Y_{2}^{\prime}+Y_{2} Y_{1}^{\prime} & 2 \bar{\Omega} Y_{1} Y_{2} \\
(2 / \bar{\Omega}) Y_{1}^{\prime} Y_{2}^{\prime} & Y_{1} Y_{2}^{\prime}+Y_{2} Y_{1}^{\prime}
\end{array}\right]\left[\begin{array}{cc}
1 & 0 \\
-2 i \bar{A} / \bar{\Omega} & 1
\end{array}\right] } \\
& \times\left[\begin{array}{cc}
Y_{1} Y_{2}^{\prime}+Y_{2} Y_{1}^{\prime} & 2 \bar{\Omega} Y_{1} Y_{2} \\
(2 / \bar{\Omega}) Y_{1}^{\prime} Y_{2}^{\prime} & Y_{1} Y_{2}^{\prime}+Y_{2} Y_{1}^{\prime}
\end{array}\right]
\end{aligned}
$$

where $Y^{\prime} \mathrm{s}$ are defined as before. The first and the third matrices on the right-hand side describe the spin motion during the two linear ramping periods, while the middle matrix is a result of the spin tune jump.

Figures 11 and 12 are the same as Figs. 8 and 9 earlier for the present crossing pattern. Figure 11 is polarization as a function of $\bar{\epsilon}$ for $\bar{A}=0.5,1,2,10$. Figure 12 shows the polarization as a function of $\bar{A}$ for $\bar{\epsilon}=0.1,0.5,1$. Note that the interference pattern is not as pronounced in this case because, unlike the previous case, there are three resonance crossings interplaying.

It is conceivable that by replacing the middle tune-jump matrix in Eq. (74) by another matrix reflecting a redefinition of the spin state, one could extend the applicability of our result approximately to the case of crossing two separate resonances whose distance between them is much larger than their resonance strengths. This direction is not explored in this report.

\section{SUMMARY}

We have developed a matrix formalism to treat spin dynamics near a single depolarization resonance in a synchrotron. It is applicable to the following operations of the synchrotron: (i) constant spin tune, constant resonance strength; (ii) sudden jumps in either the spin tune or the 

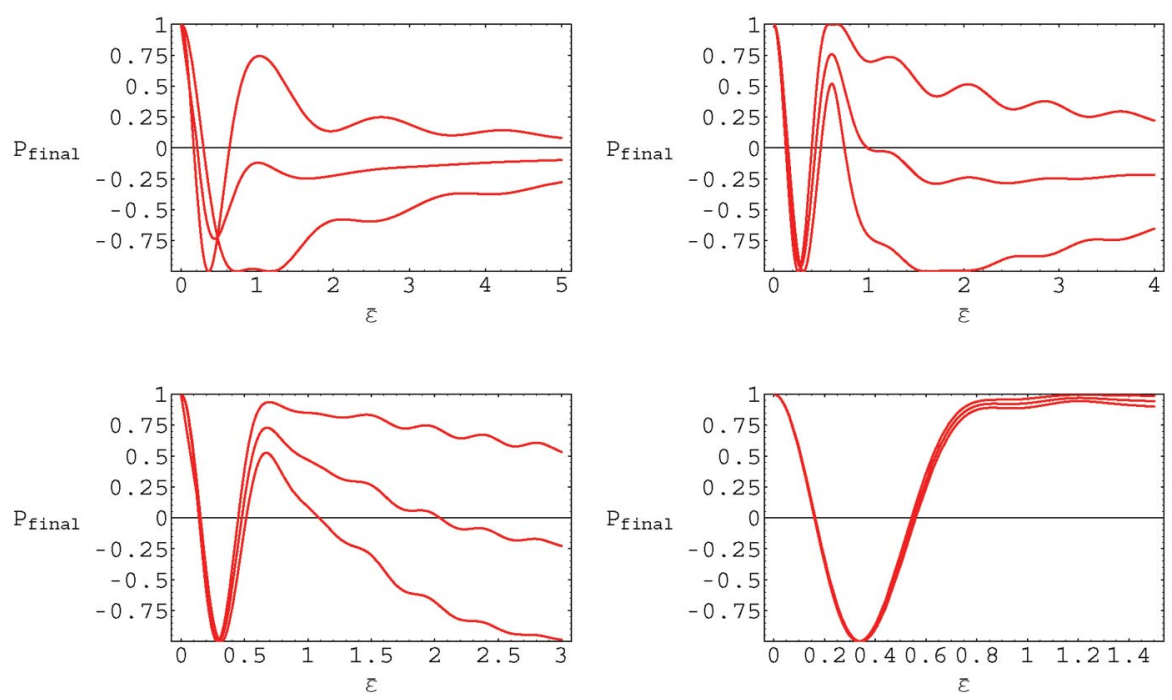

FIG. 11. (Color) Final polarization after triple-crossing a resonance in the pattern of Fig. 10 as function of $\bar{\epsilon}$ for four different values of $\bar{A}$.

resonance strength; (iii) constant resonance strength, linearly varying spin tune crossing or not crossing the resonance; (iv) any piecewise combination of the above, including repeated crossing of the resonance, each crossing having its own crossing speed.

Once a ramping procedure is given in terms of the above piecewise segments, a sequential multiplication of matrices, either in the form of $T_{\alpha, \epsilon}\left(\theta_{1}, \theta_{2}\right)$ of Eq. (18) or in the form of $U_{\Gamma, \theta_{c}, \epsilon}\left(\theta_{1}, \theta_{2}\right)$ in Eq. (52), will give the spin state at any time $\theta$.

This matrix formalism can be applied to various experimental conditions such as to extend the Froissart-Stora analysis to that with a finite spin tune change and to study interference effects of repeated resonance crossings. For a polarized beam with large energy spread, an echo phe- nomenon is suggested as a way to study these interference effects.

In the appendix, the matrix formalism is summarized in a somewhat simplified form for the case of a single particle.

\section{ACKNOWLEDGMENTS}

This work was supported by Department of Energy Contract No. DE-AC02-76SF00515. The author would like to thank Professor Alan Krisch, Professor Ernest Courant, and Professor Ronald Ruth, Dr. Vasily Morozov, Dr. Maria Leonova, and Dr. Gennady Stupakov, and colleagues in the Spin Physics Michigan-COSY Collaboration for several illuminating and enjoyable discussions.
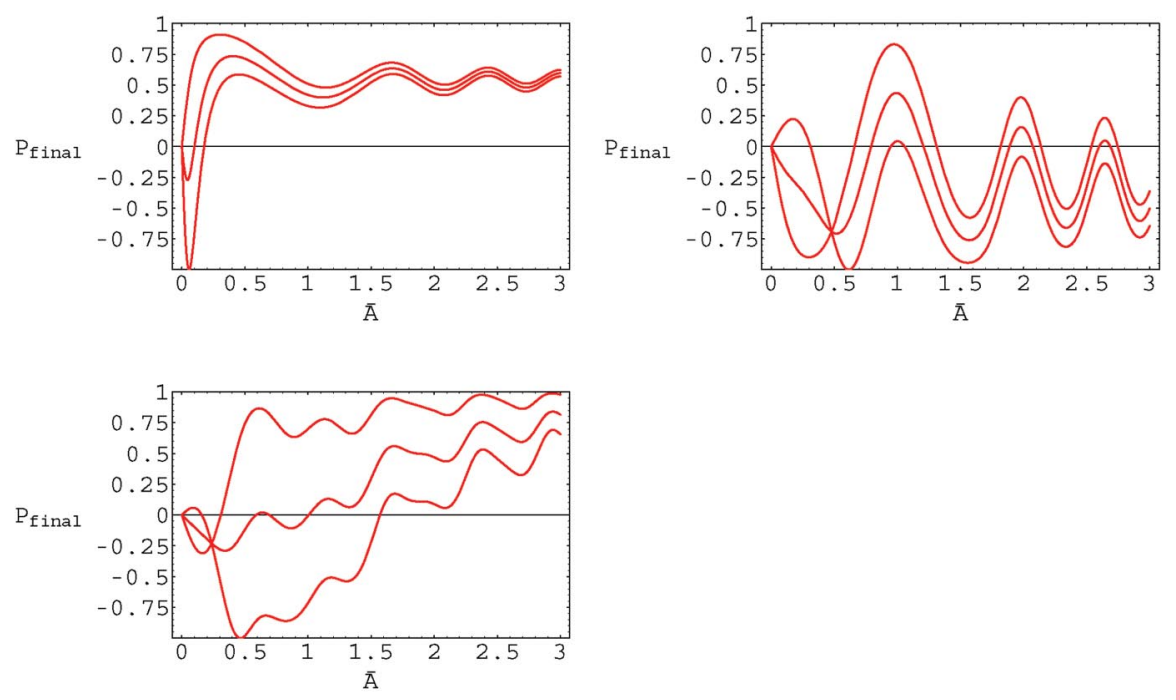

FIG. 12. (Color) Final polarization after triple crossing a resonance in the pattern of Fig. 10 as function of $\bar{A}$ for three different values of $\bar{\epsilon}$. 


\section{APPENDIX}

After the ramping procedure is completed, and the spin tune and the resonance strength are no longer changing with time, the final polarization of a single particle will in general oscillate in time according to Eq. (61) with $D_{1,2}$ given by Eq. (67) and matrix $\mathcal{M}$ given by a sequential multiplication of component matrices representing each step of crossing. For this purpose, the matrix $\mathcal{M}$ is more readily derived if we make a transformation from $(f, g)$ to $\left(\xi_{1}, \xi_{2}\right)$ by

$$
\left[\begin{array}{l}
\xi_{1} \\
\xi_{2}
\end{array}\right]=\left[\begin{array}{cc}
1 & 0 \\
i \alpha_{0} / \Omega & -i \epsilon_{0} / \Omega
\end{array}\right]\left[\begin{array}{l}
f \\
g
\end{array}\right]
$$

and the results are summarized below:

(i) With a constant resonance strength $\epsilon$ and the spin tune parked at $\alpha$, the component matrix reads

$$
\left[\begin{array}{cc}
\cos \Theta & \sin \Theta \\
-\sin \Theta & \cos \Theta
\end{array}\right]
$$

where $\Omega=\sqrt{\alpha^{2}+|\epsilon|^{2}}, \Theta=(\Omega / 2)\left(\theta_{f}-\theta_{i}\right)$, and $\theta_{i, f}$ are the initial and final times of this period.

(ii) Increasing the spin tune from below, linearly in time with slope $\Gamma>0$, the component matrix is given by

$$
\begin{aligned}
& {\left[\begin{array}{cc}
y_{1} & \bar{\Omega}_{f} y_{2} \\
\left(1 / \bar{\Omega}_{f}\right)\left(d y_{1} / d x\right) & \frac{d y_{2}}{d x}
\end{array}\right]_{x=2 \bar{A}_{f}}} \\
& \left.\times\left[\begin{array}{cc}
d y_{2} / d x & -\bar{\Omega}_{i} y_{2} \\
-\left(1 / \bar{\Omega}_{i}\right)\left(d y_{1} / d x\right) & y_{1}
\end{array}\right]\right]_{x=2 \bar{A}_{i}}
\end{aligned}
$$

where $\bar{A}_{i}=\left(\alpha_{i} / 2 \sqrt{\Gamma}\right)<0, \bar{A}_{f}=\left(\alpha_{f} / 2 \sqrt{\Gamma}\right)>0$ with $\alpha_{i, f}$ the initial and final spin tunes (relative to the exact location of the resonance $G \gamma=\kappa)$, and $\bar{\Omega}_{i, f}=\left(\Omega_{i, f} / 2 \sqrt{\Gamma}\right)=$ $\left(\sqrt{\alpha_{i, f}^{2}+|\epsilon|^{2}} / 2 \sqrt{\Gamma}\right)=\sqrt{\bar{A}_{i, f}^{2}+\bar{\epsilon}^{2}}$. The $y^{\prime}$ s are the $y_{1,2}$ functions evaluated at $x=2 \bar{A}_{i, f}$ and $a=\frac{i}{2}-\bar{\epsilon}^{2}$.

(iii) Decreasing the spin tune from above, linearly in time with slope $-\Gamma(\Gamma>0)$, the component matrix is given by

$$
\begin{aligned}
& {\left[\begin{array}{cc}
y_{1} & \bar{\Omega}_{f} y_{2} \\
\left(1 / \bar{\Omega}_{f}\right)\left(d y_{1} / d x\right) & \left(d y_{2} / d x\right)
\end{array}\right]_{x=-2 \bar{A}_{f}}} \\
& \times\left[\begin{array}{cc}
d y_{2} / d x & -\bar{\Omega}_{i} y_{2} \\
\left(-1 / \bar{\Omega}_{i}\right)\left(d y_{1} / d x\right) & y_{1}
\end{array}\right]_{x=-2 \bar{A}_{i}}
\end{aligned}
$$

now with $\bar{A}_{i}>0, \bar{A}_{f}<0$. The $y^{\prime} \mathrm{s}$ are the $y_{1,2}$ functions evaluated at $x=-2 \bar{A}_{i, f}$ and $a=-\frac{i}{2}-\bar{\epsilon}^{2}$.

(iv) A sudden jump of spin tune from $\alpha_{i}$ to $\alpha_{f}$ and synchronously in resonance strength from $\epsilon_{i}$ to $\epsilon_{f}$ contributes a matrix

$$
\left[\begin{array}{cc}
1 & 0 \\
\left(i / \Omega_{f}\right)\left[\alpha_{f}-\left(\alpha_{i} \epsilon_{f} / \epsilon_{i}\right)\right] & \epsilon_{f} / \epsilon_{i}
\end{array}\right],
$$

where $\Omega_{f}=\sqrt{\alpha_{f}^{2}+\left|\epsilon_{f}\right|^{2}}$. When $\epsilon_{f}=\epsilon_{i}$, the matrix becomes

$$
\left[\begin{array}{cc}
1 & 0 \\
\left(i / \Omega_{f}\right)\left(\alpha_{f}-\alpha_{i}\right) & 1
\end{array}\right] .
$$

When $\alpha_{f}=\alpha_{i}$, it becomes

$$
\left[\begin{array}{cc}
1 & 0 \\
\left(i \alpha_{i} / \Omega_{f}\right)\left[1-\left(\epsilon_{f} / \epsilon_{i}\right)\right] & \epsilon_{f} / \epsilon_{i}
\end{array}\right] .
$$

When $\alpha_{f}=\alpha_{i}$ and $\epsilon_{f}=0$ (the resonance is simply switched off), we have

$$
\left[\begin{array}{cc}
1 & 0 \\
i \operatorname{sgn}\left(\alpha_{i}\right) & 0
\end{array}\right]
$$

These results agree with Eqs. (28) and (29).

[1] E. D. Courant, in Possibilities for High Energy Polarized Proton Beams, AIP Conf. Proc. No. 42 (AIP, New York, 1977), p. 94.

[2] E.D. Courant and R.D. Ruth, Brookhaven National Laboratory Report No. BNL 51270, 1980.

[3] L. Teng, Fermilab Internal Report No. FN-267, 1974.

[4] S. Y. Lee, Spin Dynamics and Snakes in Synchrotrons (World Scientific, Singapore, 1997).

[5] T. Roser, Spinor Algebra, Handbook of Accelerator Physics and Engineering, edited by A. W. Chao and M. Tigner (World Scientific, Singapore, 1999).

[6] A.W. Chao, Report No. SLAC-PUB-9574, 2002, http:// www.slac.stanford.edu/ achao/lecturenotes.html

[7] Marcel Froissart and Raymond Stora, Nucl. Instrum. Methods 7, 297 (1960).

[8] See, for example, B.B. Blinov, Z.B. Etienne, A.D. Krisch, M. A. Leonova, W. Lorenzon, V.S. Morozov, C.C. Peters, V.K. Wong, and K. Yonehara, Phys. Rev. Lett. 88, 014801 (2002); V. A. Anferov, B. B. Blinov, D. Yu. Kantsyrev, A. D. Krisch, D. W. Sivers, C. M. Chu, P. Schwandt, B. von Przewoski, and V. N. Grishin, Phys. Rev. ST Accel. Beams 3, 041001 (2000); M. Bai, S. Y. Lee, J. W. Glenn, H. Huang, L. Ratner, T. Roser, M. J. Syphers, and W. van Asselt, Phys. Rev. E 56, 6002 (1997); M. Bai, L. Ahrens, J. Alessi, K. Brown, G. Bunce, P. Cameron, C. M. Chu, J.W. Glenn, H. Huang, A.E. Kponou, K. Krueger, W. Lamble, A. Luccio, Y. I. Makdisi, S. Y. Lee, M. Okamura, L. Ratner, K. Reece, T. Roser, H. Spinka, M. J. Syphers, N. Tsoupas, D. G. Underwood, W. van Asselt, N. Williams, and A. Yokosawa, Phys. Rev. Lett. 80, 4673 (1998), and experimental references quoted therein.

[9] R. D. Ruth, Report No. BNL-28325, 1980.

[10] V. Morozov (private communication).

[11] Philip M. Morse and Herman Feshbach, Methods of Theoretical Physics (McGraw-Hill, New York, 1978). The notation there is $1 F_{1}(\alpha ; \gamma ; z)=F(\alpha|\gamma| z)$.

[12] A. Krisch and V. Morozov (private communications). 